\title{
Article \\ Genome Editing of Rice eIF4G Loci Confers Partial Resistance to Rice Black-Streaked Dwarf Virus
}

\author{
Wei Wang ${ }^{1,2}$, Shuhui Ma ${ }^{1,2}$, Peng $\mathrm{Hu}^{1,3}$, Yinghua Ji ${ }^{1, *}$ and Feng Sun ${ }^{1, *}$ \\ 1 Jiangsu Key Laboratory for Food Quality and Safety-State Key Laboratory Cultivation Base, Institute of Plant \\ Protection, Jiangsu Academy of Agricultural Sciences, Nanjing 210014, China; 18256668028@163.com (W.W.); \\ m1195857235@163.com (S.M.); 15279889632@163.com (P.H.) \\ 2 College of Plant Protection, Nanjing Agricultural University, Nanjing 210095, China \\ 3 The State Key Laboratory of Crop Genetics and Germplasm Enhancement, Nanjing Agricultural University, \\ Nanjing 210095, China \\ * Correspondence: jiyinghua@jaas.ac.cn (Y.J.); sunfeng1201@126.com (F.S.)
}

Citation: Wang, W.; Ma, S.; Hu, P.; Ji, Y.; Sun, F. Genome Editing of Rice eIF4G Loci Confers Partial Resistance to Rice Black-Streaked Dwarf Virus. Viruses 2021, 13, 2100. https:// doi.org/10.3390/v13102100

Academic Editor: Feng Li

Received: 31 August 2021

Accepted: 13 October 2021

Published: 18 October 2021

Publisher's Note: MDPI stays neutral with regard to jurisdictional claims in published maps and institutional affiliations.

Copyright: ( $\odot 2021$ by the authors Licensee MDPI, Basel, Switzerland. This article is an open access article distributed under the terms and conditions of the Creative Commons Attribution (CC BY) license (https:// creativecommons.org/licenses/by/ $4.0 /)$.

\begin{abstract}
Rice black-streaked dwarf disease, caused by rice black-streaked dwarf virus (RBSDV), is a serious constraint in Chinese rice production. Breeding disease-resistant varieties through multigene aggregation is considered an effective way to control diseases, but few disease-resistant resources have been characterized thus far. To develop novel resources for resistance to RBSDV through CRISPR/Cas9-mediated genome editing, a guide RNA sequence targeting exon 1 of eIF4G was designed and cloned into a binary vector, pHUE401. This recombinant vector was used to generate mutations in the rice cultivar Nipponbare via Agrobacterium-mediated transformation. This approach produced heritable homozygous mutations in the transgene-free T1 generation. Sequence analysis of the eIF4G target region from $\mathrm{T} 1$ transgenic plants identified 3 bp deletion mutants, and analysis of the predicted amino acid sequence identified one amino acid deletion in mutants that possess near full-length eIF4G. Furthermore, our data suggest that eIF4G may plays an important role in rice normal development, as there were no eIF4G knock-out homozygous mutants in T1 generation plants. When homozygous mutant lines were inoculated with RBSDV, they exhibited enhanced tolerance to virus infection, without visibly affecting plant growth and development. However, the eif $4 g$ mutant plants showed the same sensitivity to rice stripe virus (RSV) infection as wild-type plants. Notably, the wild-type and mutant N-termini of eIF4G interacted directly with RBSDV P8 in yeast and in planta. Additionally, compared to wild-type plants, the eIF4G transcript level was reduced twofold in the mutant plants. These results indicate that site-specific mutation of rice eIF4G successfully conferred partial resistance specific to RBSDV associated with less transcription of eIF4G in mutants. Therefore, this study demonstrates that the novel eIF4G alleles generated by CRISPR/Cas9 represent valuable disease-resistant resources that can be used to develop RBSDV-resistant varieties.
\end{abstract}

Keywords: RBSDV; eIF4G; genome editing; CRISPR/Cas9; virus resistance

\section{Introduction}

Rice black-streaked dwarf virus (RBSDV), transmitted by the small brown planthopper (SBPH: Laodelphax striatellus Fallén), mainly causes rice black-streaked dwarf disease and maize rough dwarf diseases, which lead to serious cereal crop yield losses in China [1,2]. Rice plants infected by RBSDV typically exhibit acute growth abnormalities, such as severe dwarfism, dark green and stiff leaves, and tumors on leaves and stems [3-5]. Diseased rice plants generally reduce the yield by $10-40 \%$, and severely diseased rice plants even have no grains [6].

RBSDV is a double-stranded RNA virus and belongs to the genus Fijivirus within the family Reoviridae [7]. RBSDV virus particles are icosahedral, with double-layer shells containing spikes, and contain 10 double-stranded RNAs, S1-S10 [8,9]. RBSDV genomic segments encode six structural proteins P1, P2, P3, P4, P8 and P10 in its viral particle and 
seven nonstructural proteins P5-1, P5-2, P6, P7-1, P7-2, P9-1 and P9-2 [10,11]. P8, the minor core protein of the RBSDV virion, can enter the nucleus of the plant and insect cells and possesses transcriptional repression activity in tobacco suspension cells [12]. SP8 encoded by SRBSDV, a closely related Fijivirus, can interact with rice auxin response factor 17 (OsARF17) to facilitate virus infection by impairing auxin-mediated antiviral defense [13]. In addition, a recent study showed that RBSDV P8 interacts with maize AKIN $\beta$ protein (B subunit of Arabidopsis SNF1 kinase homolog in maize) and facilitates viral accumulation in maize [14]. All these results suggest that P8 plays an important role during the process of virus-host interaction.

To manage rice black-streaked dwarf disease caused by RBSDV, insecticides are frequently employed to control SBPH vectors, accompanied by increasing environmental and economic concerns [15]. Recently, genetically modified crops resistant to viruses have been shown to be an economically and environmentally feasible alternative [16,17]. Among these antiviral strategies, artificial deletion and point mutations using the clustered regularly interspersed palindromic repeats (CRISPR)/CRISPR-associated protein 9 (CRISPR/Cas9) technique in the gene encoding host factors that are essential for the virus life cycle provide virus resistance without hampering the overall health of the plant $[18,19]$. Eukaryotic translation initiation factor (eIF) genes, such as eukaryotic translation initiation factor $4 \mathrm{E}$ (eIF4E) and eukaryotic translation initiation factor $4 \mathrm{G}($ eIF $4 G)$, and their isoforms are required for RNA viruses to translate viral RNAs [20,21]. According to this knowledge, using CRISPR/Cas9 technology, sequence-specific point mutations at $e I F($ iso) $4 E$ confer complete resistance to turnip mosaic virus (TuMV) in Arabidopsis plants [22]. Similarly, the use of CRISPR/Cas9 to knock out the IF $4 E$ gene in cucumbers resulted in resistance to cucumber vein yellowing virus (CYVY), zucchini yellow mosaic virus (ZYMV) and papaya ring spot mosaic virus-W (PRSV-W) under greenhouse conditions [23]. In rice plants, novel eIF4G alleles produced through the CRISPR/Cas9 system confer resistance to rice tungro spherical virus (RTSV) [24]. Thus, the novel eIF4E/eIF4G alleles generated by CRISPR/Cas9-mediated genome editing are expected to be used in the development of virus-resistant crop plants.

In this study, to introduce novel alleles of rice eIF4G loci, we mutated eIF4G in Oryza sativa var. japonica $c v$. Nipponbare using CRISPR/Cas9-mediated genome editing technology and generated the T0 transgenic rice plant that carried eIF4G mutants. The selected $\mathrm{T} 2$ rice plants that carried an edited $e I F 4 G$ gene but not the introduced transgene were tested for resistance to RBSDV. Compared with wild-type plants, homozygous rice plants with edited eIF4G genes had significantly increased tolerance to RBSDV, without affecting rice stripe virus (RSV) infection and normal plant growth. Furthermore, the wild-type and mutant N-termini of eIF4G interacted directly with RBSDV P8 in yeast and in planta. Altogether, this study provides important information on the mechanism of interaction between RBSDV and rice plants, and novel eIF4G alleles represent valuable materials for the development of RBSDV-resistant varieties.

\section{Materials and Methods}

\subsection{Sources of Virus, Vectors, and Plant Materials}

RBSDV or RSV-infected rice plants were obtained from fields in Jiangsu Province, China, and the virus was confirmed by RT-PCR assay [4]. The nonviruliferous small brown planthopper (SBPH) strains were collected from fields in Jiangsu Province, China, and reared on rice seedlings (Oryza sativa L. cv. Wuyujing No. 3) in glass incubators in an insectrearing room at $25^{\circ} \mathrm{C}$. The rice cultivar Nipponbare (O. sativa L. subsp. japonica, cv. NIP) was used as a background to generate eIF4G CRISPR/Cas9 mutant (eif4g) lines. All rice plants were grown in the greenhouse at $28-30{ }^{\circ} \mathrm{C}$ with a $12 \mathrm{~h}$ light $/ 12 \mathrm{~h}$ dark photoperiod.

\subsection{Viruses Inoculation Assay}

Nonviruliferous instar nymphs of SBPHs were fed on RBSDV(or RSV)-infected rice plants for 7 days to acquire the virus. Then, the nymphs were transferred to healthy rice 
seedlings and allowed to feed for 10 days. The percentage of viruliferous SBPHs was determined by dot enzyme-linked immunosorbent assay (Dot-ELISA) using the monoclonal antiRBSDV or antiRSV antibody [25]. Transgenic and wild-type rice plants at the 2 two-leaf-stage were inoculated with 2 viruliferous SBPHs per plant and were kept in a glass incubator containing 30 plants. SBPHs were moved after a 2-day inoculation access period. The inoculated seedlings were transplanted into an experimental field at the Jiangsu Academy of Agricultural Sciences for symptom development, and nonviruliferous insects were used for mock inoculation.

\subsection{Evaluation of RBSDV or RSV Resistance}

The RBSDV or RSV inoculated rice seedlings were transplanted into an experimental plot at the Jiangsu Academy of Agricultural Sciences, Nanjing, China. The seedlings were grown under a standard agricultural practice without the using of pesticides during growth. Four weeks after transplanting ( $28 \mathrm{dpi}$ ), the RBSDV or RSV incidence was recorded for the wild-type and its mutant lines, respectively. Three replicates were used for each treatment, and each replicate contained at least 30 rice seedlings, and the disease incidence of each rice line (\%) was determined use the following formula: number of RBSDV (or RSV)-infected plants/total number of plants counted $\times 100$. In addition, 10 rice seedlings were pooled at 28 dpi for qRT-PCR and Western blot analysis.

\subsection{DNA Constructs and Transgenic Plants}

The N-terminus of wild-type eIF4G (eIF4G-N) or mutant eIF4G (eIF4G-N"Nu) and fulllength RBSDV P8 sequences were amplified by PCR from RBSDV-infected rice plants, and the PCR products were cloned individually into $\mathrm{pDONR}$-zero vectors using BP Clonase Enzyme Mix (Invitrogen, Carlsbad, CA, USA). The eIF4G-N or eIF4G-N ${ }^{\mathrm{mu}}$ and P8 sequences were then transferred to the appropriate destination vectors using LR Clonase (Invitrogen) for yeast two-hybrid and luciferase complementary imaging assays.

For gene editing, rice eIF4G-specific sgRNA was designed by the CRISPR PLANT website (http:/ / www.genome.arizona.edu/crispr/CRISPRsearch.html, accessed on 6 October 2018). These sgRNA oligos were synthesized (Genscript, Nanjing, China) and cloned into the pHUE401 vector by Golden Gate cloning, as previously described [26]. CRISPR/Cas9 vectors were introduced into Agrobacterium tumefaciens strain GV3101 by electroporation and transformed into Nipponbare by Agrobacterium-mediated transformation. The primers used in this study are listed in Supplementary Table S1.

\subsection{Western Blot Assay}

Western blotting was performed according to the protocol described previously [27]. Briefly, rice total proteins were extracted in $2 \times$ SDS-loading buffer $(100 \mathrm{mM}$ Tris- $\mathrm{HCl}(\mathrm{pH}$ 6.8), $4 \%$ SDS, $0.2 \%$ bromophenol blue, $20 \%$ glycerol, $2 \%$ beta-mercaptoethanol), separated by $10 \%$ SDS-PAGE and transferred onto PVDF membranes. The membranes were blocked and inoculated with an antiRBSDV P6 or antiRSV SP (provided by Jianxiang Wu, Zhejiang University, Hangzhou, China) or antiactin (Enogene, Nanjing, China) or antiRubisco (Sangon Biotech, Shanghai, China) antibody for $2 \mathrm{~h}$ at room temperature. An HRP-conjugated antirabbit antibody was used as the secondary antibody (Abmart, Shanghai, China). Signals were developed in ECL buffer (Vazyme Biotech, Nanjing, China) and recorded with a Tanon 5200 Luminescent Imaging Workstation (Tanon, Shanghai, China).

\subsection{Quantitative Reverse-Transcription PCR ( $q R T-P C R)$}

Total RNA was extracted from $100 \mathrm{mg}$ rice plants with RNAiso Plus reagent (Takara, Dalian, China) according to the manufacturer's instructions. cDNA was synthesized with the iScript ${ }^{\mathrm{TM}}$ cDNA Synthesis Kit (Bio-Rad, Hercules, CA, USA) using random hexamers as primers. qPCR was performed using SsoFast EvaGreen Supermix (Bio-Rad, Hercules, CA, USA) on a Bio-Rad iQ5 qRT-PCR system with gene-specific primers (Supplementary 
Table S1). The results were normalized to reference gene expression (UBQ10) using the $2^{-\Delta \Delta \mathrm{Ct}}$ method reported previously [28].

\subsection{Yeast Two-Hybrid Assay}

The Matchmaker Gold Y2H system (Clontech, Mountain View, CA, USA) was used to identify interactions between eIF4G-N (or eIF4G-N ${ }^{\mathrm{mu}}$ ) and RBSDV P8. The CDSs of eIF4G$\mathrm{N}$ (1-1053) (or eIF4G-N ${ }^{\mathrm{mu}}$ ) and RBSDV P8 were cloned into pGBK-DC and pGAD-DC gateway destination vectors (provided by Xiuren Zhang, Texas A\&M University, College Station, USA), respectively, by the LR reaction (Invitrogen, Carlsbad, CA, USA). The plasmids pGBK-eIF4G-N (or pGBK-eIF4G-N ${ }^{\mathrm{mu}}$ ) and pGAD-P8 were cotransformed into the Y2H Gold (Clontech) yeast strain and selected on medium lacking leucine, tryptophan, histidine and adenine. The plasmids PGBK- 53 and pGAD-T7 were cotransformed into the $\mathrm{Y} 2 \mathrm{H}$ Gold yeast strain as a positive control.

\subsection{Luciferase Complementary Imaging Assay (LCI Assay)}

The CDSs of eIF4G-N (1-1053) (or eIF4G-N ${ }^{\mathrm{mu}}$ ) and RBSDV P8 were cloned into pCAMBIA-DC-NLuc and pCAMBIA-CLuc-DC (provided by Xiuren Zhang, Texas A\&M University, College Station, USA), respectively, by the LR reaction. Then, the constructs were transformed into $A$. tumefaciens strain GV3101. Agrobacteria harboring the pCAMBIAeIF4G-N-Nluc and pCAMBIA-CLuc-P8 plasmids co-infiltrated into 4-week-old N. benthamiana leaves. After 2 days, the infiltrated leaves were sprayed with $10 \mathrm{mM}$ luciferin (Promega, Madison, WI, USA) and photographed using the Tanon 5200 Luminescent Imaging Workstation (Tanon, Shanghai, China).

\section{Results}

\subsection{Generation and Characterization of Genome-Edited eIF4G Rice Plants}

To investigate the function of rice eIF4G loci in RBSDV infection, we aimed to use CRISPR/Cas9-mediated targeted genome editing of eIF4G (LOC_Os07g36940) to develop RBSDV-resistant rice plants. To design sgRNAs, we identified eIF4G target sgRNA sequences using CRISPR PLANT tools (http:/ / www.genome.arizona.edu/crispr/CRISPRsearch.html, accessed on 6 October 2018). sgRNAs targeting the first exon with the Bsg I restriction site were used for genome editing (Figure 1A). We cloned this sgRNA into a binary vector (pHUE401) [26], and this pHUE401-eIF4G CRISPR/Cas9 construct was introduced into rice plants (Nipponbare) by Agrobacterium-mediated transformation.

Ten rice plants were generated by hygromycin selection and analyzed for CRISPR/Cas9induced mutation in eIF4G by restriction enzyme digestion of a PCR fragment encompassing the target region (Figure 1B). These results showed that $50 \%$ of the T0 transgenic

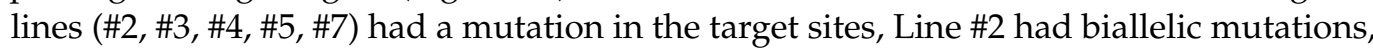
and the others had monoallelic mutations. We cloned and sequenced the PCR fragments from T1 generation plants of two lines (\#2 and \#5) and found that three nucleotides were deleted from the target sequences, as shown in Figure 1C. Analysis of the translated protein sequence identified that 79-site proline were deleted in the eIF4G protein in the two mutant lines (\#2 and \#5) (Figure 1C). To determine the effect of mutants on the eIF4G expression level, we analyzed the eIF4G mRNA transcription level by qRT-PCR assay using homozygous mutant lines (\#2 and \#5). The results showed that, compared with wild-type (WT) plants (Nipponbare), the expression of eIF4G RNA transcript was significantly reduced in the mutant plants eif4g \#2 and eif4g \#5 (Figure 1D). 


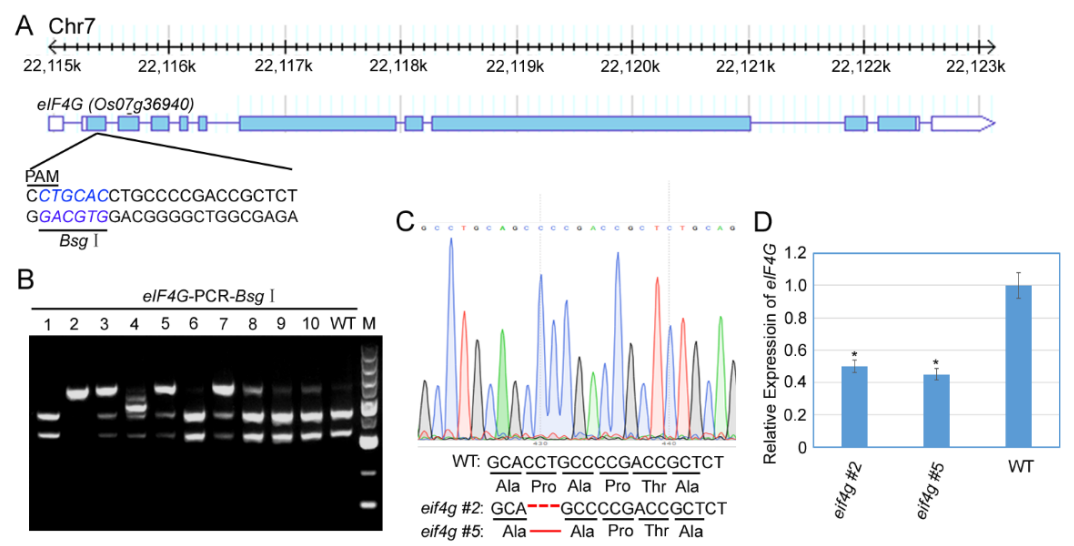

Figure 1. Genomic editing of rice $e I F 4 G$ by CRISPR/Cas9. (A) Schematic diagram of the CRISPR/Cas9 target sites in $e I F 4 G$. The sgRNA target sequences are shown in black letters, and Bsg I digestion sequences are shown in blue letters. (B) Mutation analysis of $10 \mathrm{~T} 0$ transgenic lines by Bsg I digestion of PCR fragments. (C) DNA and amino acid sequence analysis of mutated alleles identified from cloned PCR fragments of two representative T1 transgenic lines. DNA and amino acid deletions are denoted by red dashes. (D) qRT-PCR analysis of eIF4G mRNA transcription levels in mutants and WT rice plants. Signal intensities for each transcript were normalized to those for UBQ. All data are means $\pm \mathrm{SD}(n=3)$. The statistical significance of the difference between the eif $4 g$ mutant lines and wild-type plants was determined using Student's $t$-test. ${ }^{*} p<0.05$.

\subsection{Segregation of Mutation and cas9 from the Transgene in the T1 Generation}

Among the T0 generation of eif $4 \mathrm{~g}$ mutants, we selected the \#2, \#4 and \#5 plants from which to generate T1 lines. First, we tested the presence of transgenes by PCR with Cas9specific primers within T1 plants from eif4g \#2, which was a biallelic mutant in the T0 generation. Among 9 plants derived from eif $4 \mathrm{~g}$ \#2, 6 carried Cas9 transgenes $(1,2,3,5,6,9)$, and $3 \mathrm{did}$ not $(4,7,8)$ (Figure $2 \mathrm{~A})$. Sequencing of amplicons of the target region showed that all T1 generations of eif $4 g$ \#2 were homozygous mutants. These results indicated that the transgene-free T1 generation of eif $4 \mathrm{~g} \# 2$ plants $(4,7,8)$ were all homozygous mutants.

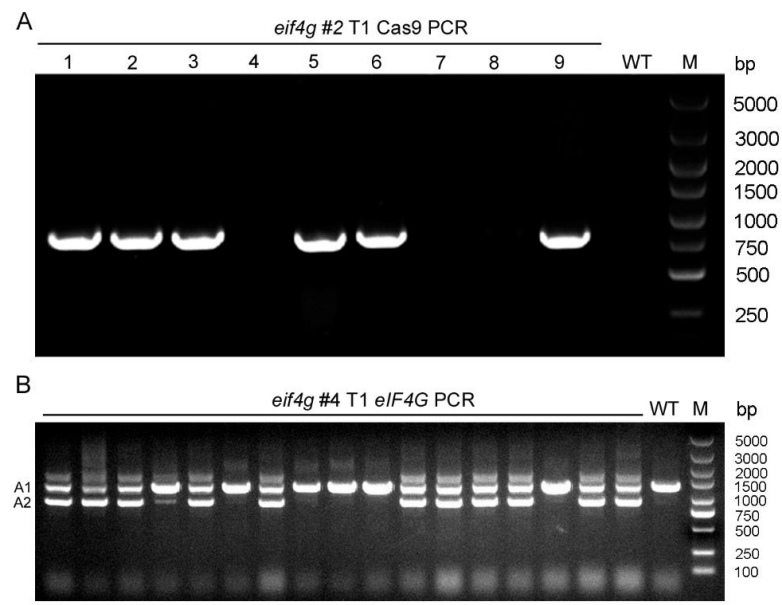

\footnotetext{
WT:gggctgtgagccaacccgacacgcacggettccagcctgcacctgccccgaccgctctgcagacgccg A1:gggctgtgagccaacccgacacgcacggcttccagcctgca---gccccgaccgctctgcagacgccg A2: gggttggctcacagccctctggttgtggggcaatgg-437 deletion
}

Figure 2. Transgene cas 9 and mutation screening in T1 generation rice plants. (A) Transgene screening of eIF4G-targeted T1 transgenic plants by genomic DNA PCR. WT: wild-type plants. M: DNA marker. (B) eif4g mutant screening in eIF4G-targeted T1 transgenic plants (\#4) by genomic DNA PCR. WT: wildtype plants; DNA sequence deletions are denoted by black dashes, and DNA sequence insertions are denoted by blue letters. The sgRNA target sequences are shown in red letters. M: DNA marker. 
For the T0 generation of eif4g \#4 mutants containing eIF4G small fragments (A2), we analyzed these $e I F 4 G$ small fragments in the T1 population plants with PCR. Our results showed that there were no eIF4G small fragment homozygous mutants in the T1 generation of eif4g \#4 (Figure 2B). DNA sequencing of eIF4G small fragments showed that this mutant carried a $33 \mathrm{bp}$ insertion and $437 \mathrm{bp}$ deletion (Figure 2B). These results indicate that $e I F 4 G$ plays an important role in rice plant development and that knocking out eIF4G may be lethal.

\section{3. eIF4G Edited Rice Plants Confer Partial Resistance to RBSDV}

To examine whether these eif4g mutant rice plants edited by CRISPR/Cas9 affect RBSDV resistance, two homozygous T2 populations (eif4g \#2 and eif4g \#5) and wildtype plants were inoculated with RBSDV. The wild-type plants showed typical RBSDV symptoms at 21 days postinoculation (DPI), including severe dwarfing and dark green leaves. Both eif4g mutant rice lines showed mild symptoms (Figure 3A,B). At $28 \mathrm{dpi}$, the RBSDV disease incidence of eif $4 g$ mutant lines (eif4g \#2 and eif4g \#5) was significantly lower than the RBSDV disease incidence of the wild-type plants (Figure 3C). To confirm RBSDV virus infection, we analyzed viral RNA accumulation by qRT-PCR using RBSDV P9-1 gene-specific primers and virus P6 protein accumulation by Western blot assay. The results showed that compared with wild-type plants, the accumulation levels of RBSDV P9-1 RNA in the eif4g mutant lines were significantly decreased (Figure 3D). Similarly, the eif4g mutant lines accumulated less P6 protein than wild-type plants at 21 dpi (Figure 3E). These results all showed that the eIF4G-edited lines conferred partial resistance to RBSDV.
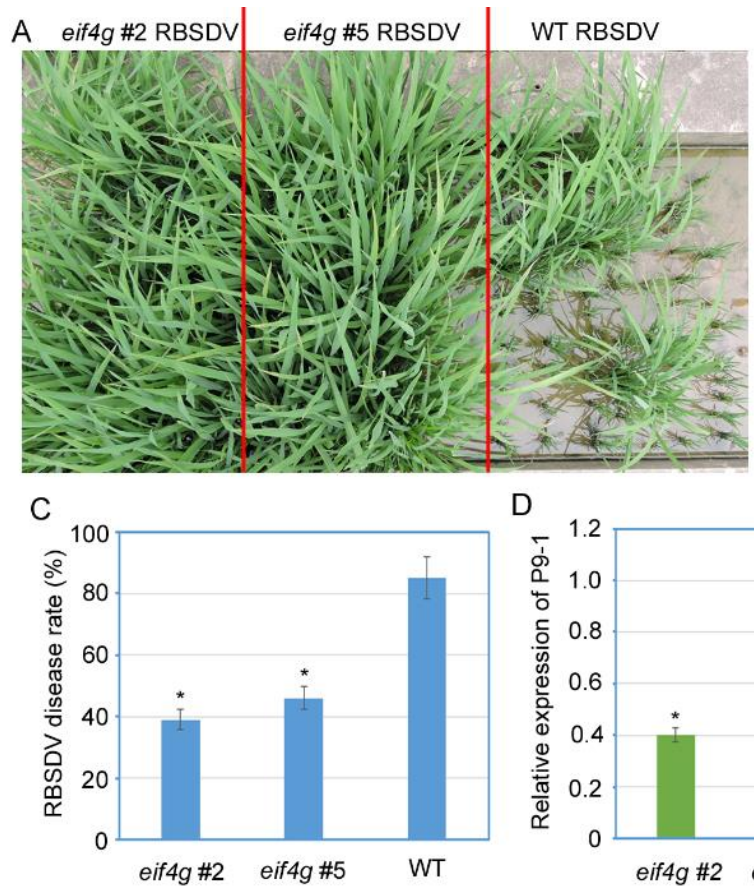

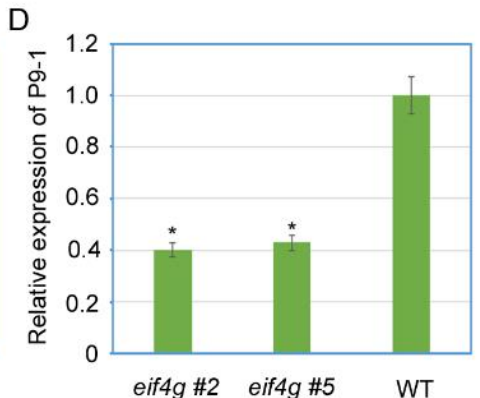

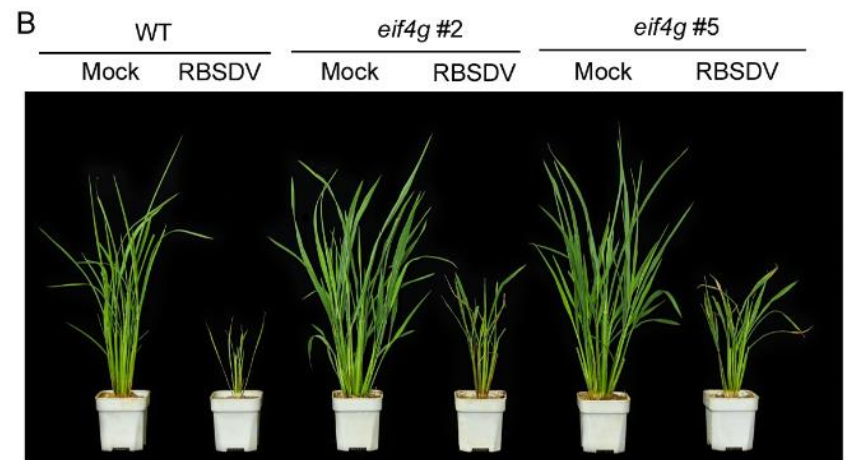

E

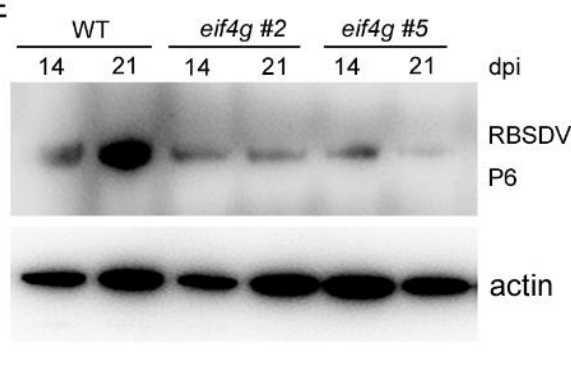

Figure 3. Evaluation of rice black-streaked dwarf virus (RBSDV) resistance of rice eif $4 g$ mutant lines. (A,B) Disease symptoms of mock-inoculated and RBSDV-infected eif4g mutant lines (eif4g \#2, eif4g \#5) and wild-type (WT, Nipponbare) rice plants. Photographs were taken at 28 days postinoculation (dpi). (C) Incidence of RBSDV disease rate in eif $4 g$ mutant lines (eif4g \#2, eif4g \#5) and wild-type (WT, Nipponbare) rice plants at 28 dpi. (D) qRT-PCR analysis of RBSDV P9-1 mRNA transcription levels in eif $4 g$ mutant lines (eif4g \#2, eif $4 g$ \#5) and WT rice plants at $28 \mathrm{dpi}$. Signal intensities for each transcript were normalized to the signal intensity for UBQ. (E) Western blot analysis of RBSDV-encoded P6 protein accumulation in virus-infected eif4g mutant lines (eif4g \#2, eif4g \#5) and wild-type (WT, Nipponbare) rice plants using a P6-specific antibody. The actin protein level served as a loading control. Rice plants were all collected at 28 dpi. All data are means \pm SD $(n=3)$.The statistical significance of the difference between the eif4g mutant lines and wild-type plants was determined using Student's $t$-test. ${ }^{*} p<0.05$. 
To determine if the eif $4 \mathrm{~g}$ mutant rice plants have a broader spectrum of resistance to virus infection, these mutant plants were inoculated with rice stripe virus (RSV), which is type species in the genus Tenuivirus and transmitted by small brown planthopper (SBPH). The RSV infection assay showed that the eif4g mutant rice plants developed severe stunting symptom similar to wild-type plants (Figure S1A). At $28 \mathrm{dpi}$, the RSV disease incidence of eif4g mutant lines (eif4g \#2 and eif4g \#5) was also similar to wild-type plants (Figure S1B). qRT-PCR and Western blot results both showed that RSV SP mRNA and proteins accumulated in eif4g mutant plants were comparable with wild-type plants (Figure S1C,D). These data indicated that the eif $4 \mathrm{~g}$ mutant plants had no effect on RSV tolerance.

To clarify the effect of eif $4 \mathrm{~g}$ mutants on plant growth and development, agronomic parameters such as plant height, weight of 1000 grains, tiller number per plant and panicle length were measured in two eif $4 g$ mutant lines and wild-type plants. Compared to wildtype plants, eif $4 \mathrm{~g}$ mutants exhibited similar agronomic parameters but a significant increase in panicle length (Figure 4).
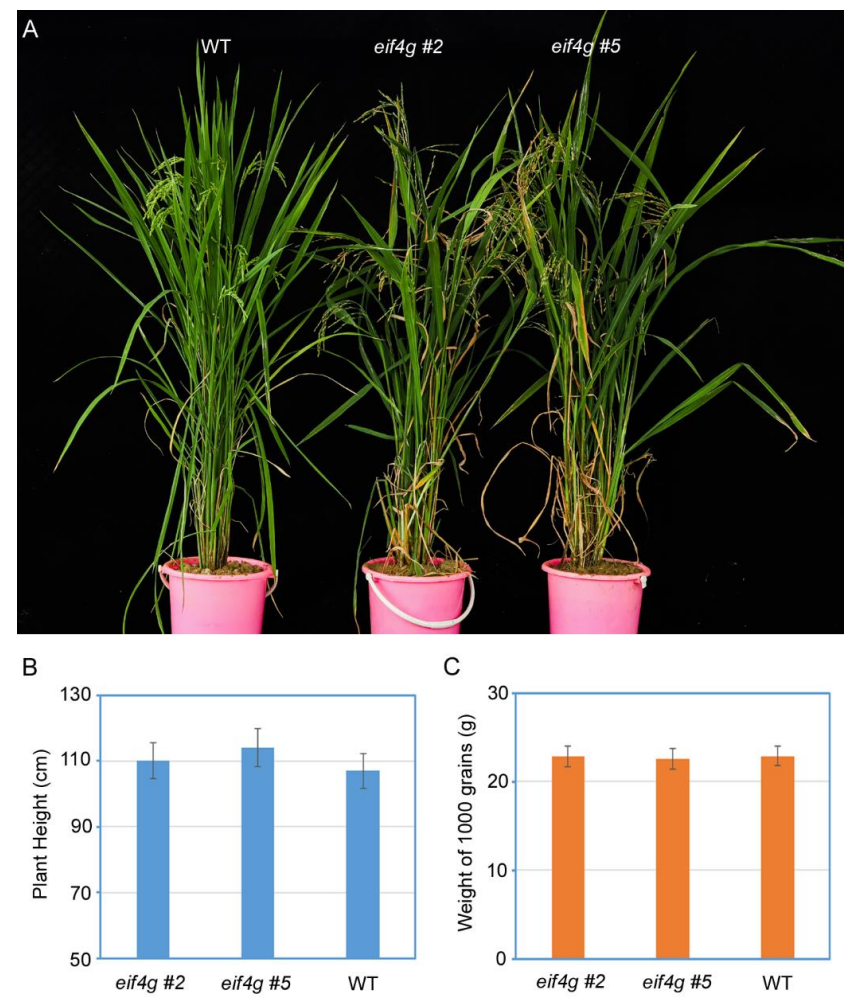

E
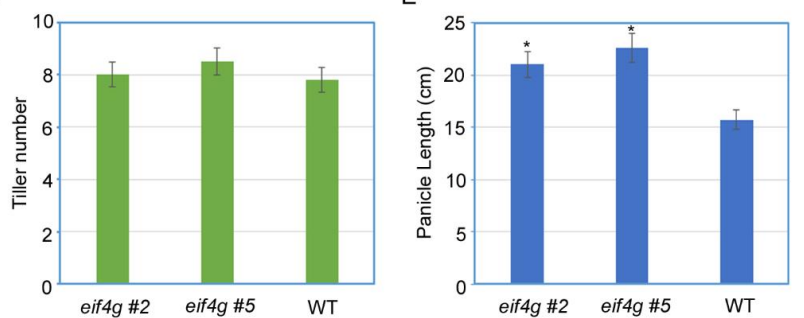

Figure 4. Examination of the agronomic parameters of rice eif4g mutant lines. (A) Development phenotype of eif $4 g$ mutant lines (eif $4 g$ \#2, eif $4 g$ \#5) and wild-type (WT, Nipponbare) rice plants. Photographs were taken at 60 days after germination. (B-E) The agronomic parameters of eif $4 g$ mutant lines (eif4g \#2, eif $4 g$ \#5) and wild-type (WT, Nipponbare) rice plants. All data are means \pm SD $(n=20)$.The statistical significance of the difference between the eif $4 g$ mutant lines and wild-type plants was determined using Student's $t$-test. ${ }^{*} p<0.05$. 


\subsection{The N-Terminus of eIF4G Directly Interacted with RBSDV P8 in Yeast and Plants}

To understand the molecular mechanism of eIF4G-mediated RBSDV resistance, we used a yeast two-hybrid assay to screen nine RBSDV proteins (P5-1, P5-2, P6, P7-1, P7-2, P8, P9-1, P9-2 and P10) for eIF4G interactions using the N-terminus of eIF4G (eIF4G-N) fused to the GAL4 DNA binding domain (BK-eIF4G-N) as bait. The yeast two-hybrid results indicated that cotransformation with BK-eIF4G-N and AD-P8 vectors allowed yeast to grow on selective media without histidine and adenine, in contrast to the negative controls (Figure 5A). The interaction was also detected by analyzing LacZ reporter gene activation. This result indicates a direct interaction between eIF4G-N and P8. To evaluate this interaction in planta, luciferase complementary imaging (LCI) assays were performed. In LCI experiments, the $\mathrm{N}$ - and C-terminal parts of luciferase (nLuc and cLuc) were fused to eIF4G-N and P8, respectively, and were coexpressed in Nicotiana benthamiana leaves by Agrobacterium-mediated infiltration. In our LCI assay, coexpression of eIF4G-N-nLuc and P8-cLuc restored the luciferase catalytic activity, suggesting that P8 was physically close to the eIF4G protein in vivo (Figure 5B). To further examine whether P8 interacted with the eIF4G mutant protein (eIF4 $\mathrm{G}^{\mathrm{mu}}$ ) generated by CRISPR/Cas9, we conducted yeast two-hybrid and LCI assays. The results of Figure 5 show that the eIF4G mutant did not affect the interaction with the RBSDV P8 protein. Together, these assays clearly indicated that eIF4G-N and P8 interact in vivo.

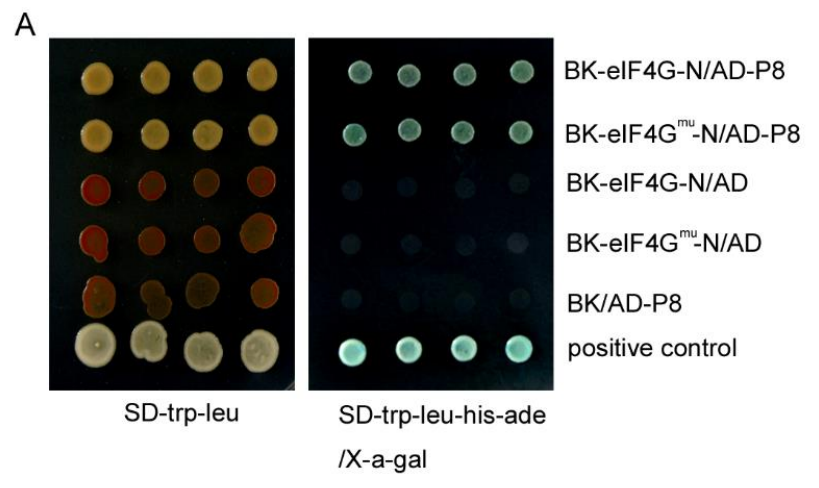

B
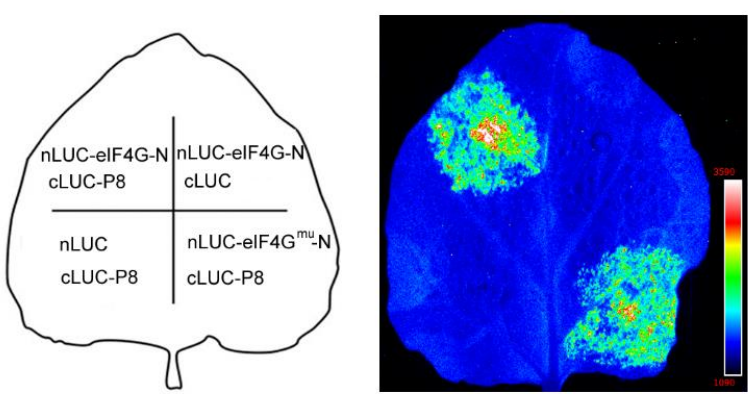

Figure 5. N-terminus of eIF4G (eIF4G-N) interacts with RBSDV P8 in yeast and in planta. (A) Yeast two-hybrid assays show that eIF4G-N interacts with the RBSDV P8 protein. The plasmid combinations indicated on the right side of the image panel were cotransformed into yeast strain $\mathrm{Y} 2 \mathrm{H}$ Gold, and the yeast cells were spotted on SD/-Trp/-Leu and SD/-Trp/-Leu/-His/-Ade selective medium with X-a-gal and grown at $30^{\circ} \mathrm{C}$ for 3-4 d. Positive control, full-length pGADT7-53 and pGBKT7-T7. (B) The luciferase complementation imaging assay indicates that eIF4G-N interacts with the RBSDV P8 protein in planta. The plasmid combinations indicated on the left side of the image panel were coexpression Nicotiana benthamiana leaves by Agrobacterium-mediated infiltration. After $48 \mathrm{~h}, \mathrm{~N}$. benthamiana leaves were sprayed with Lucifer, and a fluorescence signal appeared where eIF4G-N-nLuc or eIF4G $\mathrm{G}^{\mathrm{mu}}$-N-nLuc was coexpressed with cLuc-P8. eIF4G ${ }^{\mathrm{mu}}$, eIF4G mutant protein generated by CRISPR/Cas9. 


\section{Discussion}

RBSDV causes rice black-streaked dwarf disease, which occurs in East Asian countries and is responsible for severe rice yield losses [29]. To manage rice RBSDV disease, in the absence of commercially resistant varieties, the application of insecticides against $\mathrm{SPBH}$ vectors is the most common approach. However, this approach has polluted the environment and led to the development of insecticide-resistant SBPHs [30]. Therefore, genetic resistance for disease control is the most sustainable and environmentally friendly control strategy [31]. CRISPR/Cas9 has become a powerful tool for developing resistance resources against virus infection in various plant species [32]. In our study, we developed CRISPR/Cas9-edited rice eif4g mutants through Agrobacterium-mediated transformation. Sequencing of the $e I F 4 G$ target region revealed that indels occurred 3 bp upstream of the PAM site, creating a 3-bp deletion or 1 amino acid deletion (Figure 1C). Notably, we obtained two homozygous eif4g mutant lines in the T2 generation, which shared the same mutant type and were free of the Cas9 transgene (Figure 1C). These two eif4g mutant lines confer RBSDV partial resistance but without any growth and development defects (Figures 3 and 4). Thus, in this study, the RBSDV-tolerance plants generated by CRISPR/Cas9 may represent a valuable resistance material and improve RBSDV resistance by molecular rice breeding.

Plant viruses, obligate parasites, recruit cellular translation factors to synthesize their proteins, regulate their replication and potentiate their local and systemic movement. Many natural plant virus recessive resistance genes have been targeted to translation factors eIF4E and eIF4G or their isoforms, eIFiso4E and eIFiso4G [33]. Editing or knockout of one of these factors confers virus resistance but does not interfere with the overall health of the plant. Previous studies have shown that different potyviruses selectively recruit eIF4G or its isoforms to accomplish their infectious cycles. In Arabidopsis plants, the eIF4G knockout mutant is resistant to clover yellow vein virus (ClYVV), the eIF(iso)4G1 mutant is resistant to plum pox virus (PPV) and lettuce mosaic virus (LMV), and the eIF(iso)4G1 eIF(iso)4G2 double mutant is resistant to turnip mosaic virus (TuMV) [34]. Similarly, Arabidopsis homozygous for T-DNA insertions in the eIF4G gene displays reduced multiplication of cucumber mosaic virus (CMV) and turnip crinkle virus (TCV) [35]. In rice plants, the natural resistance gene to rice yellow mottle virus (RYMV) is correlated with mutations in eIF(iso)4G [36], and novel eIF4G alleles generated using CRISPR/Cas9 result in resistance to rice tungro spherical virus (RTSV) [24]. Here, we show that new rice eIF4G alleles generated by CRISPR/Cas9 technology confer RBSDV partial resistance without hindering plant growth and affecting RSV infection (Figure S1). These data suggest that RBSDV and RSV recruit different translation initiation factors to complete virus life cycles. Nevertheless, the resistance level to RBSDV was not completely immune (Figures 3 and 4), which can be explained because RBSDV may use eIF(iso) $4 \mathrm{G}$ for virus replication and systemic movement and therefore can cause mild symptoms. RBSDV resistance genetic analysis also demonstrates that several quantitative trait loci (QTLs) have been identified in rice genetic populations $[37,38]$. These results suggest that RBSDV may recruit eIF4G and eIF(iso)4G to complete its disease cycle. Thus, eIF4G and eIF(iso) $4 \mathrm{G}$ double-mutant plants would be expected to show more resistance to RBSDV.

Previous studies of potyviruses suggest that mutations in the eIF4E/(iso)4E protein interrupt the interaction between viral genome-linked protein (VPg) and eIF4E/(iso)4E and confer plant resistance to virus infection $[39,40]$. The VPg of potato virus A (PVA), as a cap-like structure, recruits host eIF(iso)4E via a specific motif for PVA RNA stabilization, as well as viral protein synthesis [41]. Similarly, rice yellow mottle virus (RYMV) VPg interacts with the central domain of rice eIF(iso)4G, and the insertion of the E309K mutation in eIF(iso) $4 \mathrm{G}$ confers resistance in plants, diminishing the interaction with VPg [42]. In this study, we demonstrated a direct interaction between RBSDV P8 and the N-terminal domain of rice eIF4G both in yeast and in planta using yeast two-hybrid and luciferase complementary imaging (LCI) assays (Figure 5). Deletion of the 79-site proline mutation in eIF4G generated via CRISPR/Cas9, which confers partial resistance to RBSDV in plants, 
did not diminish the interaction with P8 (Figure 5). These results suggest that RBSDV P8 may recruit rice eIF4G to facilitate virus infection, and the conferred partial resistance to viruses of eif $4 \mathrm{~g}$ mutant plants may be due to the decreased transcriptional level of eIF4G in mutant plants (Figure 1E). Additional experiments are needed to reveal the precise relationship between rice $e I F 4 G$ and RBSDV proteins and whether the eIF4G-P8 interaction functions as a convergence node of the viral cycle.

Supplementary Materials: The following are available online at https://www.mdpi.com/article/10 $.3390 / v 13102100 /$ s1, Figure S1. Evaluation of rice stripe virus (RSV) resistance of rice eif4g mutant lines, Table S1: Primers sequences used for cloning of transgenes and testing the viral infection.

Author Contributions: F.S. and Y.J. conceived and designed the experiments. W.W., S.M. and P.H. conducted the experiments. F.S. and Y.J. analyzed the data. F.S. and W.W. wrote the manuscript. All authors read and approved the manuscript.

Funding: This research was supported by Jiangsu Agriculture Science and Technology Innovation Fund [No. CX (21)1011].

Institutional Review Board Statement: Not applicable.

Informed Consent Statement: Not applicable.

Data Availability Statement: Not applicable.

Acknowledgments: We are grateful Xiuren Zhang at Texas A\&M University for providing yeast two-hybrid and LCI assay vectors and Jianxiang Wu at Zhejiang University, China for providing the RBSDV P6 and RSV SP antibody.

Conflicts of Interest: The authors declare no conflict of interest.

\section{References}

1. Shikata, E.; Kitagawa, Y. Rice black-streaked dwarf virus: Its properties, morphology and intracellular localization. Virology 1977, 77, 826-842. [CrossRef]

2. Zhou, Y.; Zhang, L.; Zhang, X.; Zu, H.; Di, H.; Dong, L.; Liu, X.; Zeng, X.; Weng, J.; Wang, Z.; et al. Rice black-streaked dwarf virus Genome in China: Diversification, Phylogeny, and Selection. Plant Dis. 2017, 101, 1588-1596. [CrossRef] [PubMed]

3. Sun, F.; Yuan, X.; Xu, Q.; Zhou, T.; Fan, Y.; Zhou, Y. Overexpression of Rice Black-Streaked Dwarf Virus P7-1 in Arabidopsis Results in Male Sterility Due to Non-Dehiscent Anthers. PLoS ONE 2013, 8, e79514. [CrossRef] [PubMed]

4. Zhao, C.; Sun, F.; Li, X.; Lan, Y.; Du, L.; Zhou, T.; Zhou, Y. Reverse transcription-recombinase polymerase amplification combined with lateral flow strip for detection of rice black-streaked dwarf virus in plants. J. Virol. Methods 2019, 263, 96-100. [CrossRef] [PubMed]

5. Wu, N.; Zhang, L.; Ren, Y.; Wang, X. Rice black-streaked dwarf virus: From multiparty interactions among plant-virus-vector to intermittent epidemics. Mol. Plant Pathol. 2020, 21, 1007-1019. [CrossRef]

6. Sun, F.; Xu, Q.; Cheng, Z.; Fan, Y.; Zhou, Y. Advances in rice black-streaked dwarf disease in China. Jiangsu J. Agric. Sci. 2013, 29, 195-201.

7. Wang, Z.-H.; Fang, S.-G.; Xu, J.-L.; Sun, L.-Y.; Li, D.W.; Yu, J.-L. Sequence Analysis of the Complete Genome of Rice Black-Streaked Dwarf Virus Isolated from Maize with Rough Dwarf Disease. Virus Genes 2003, 27, 163-168. [CrossRef]

8. Milne, R.G.; Conti, M.; Lisa, V. Partial purification, structure and infectivity of complete maize rough dwarf virus particles. Virology 1973, 53, 130-141. [CrossRef]

9. Milne, R.G.; del Vas, M.; Harding, R.M.; Marzachi, R.; Mertens, P.P.C. Genus Fijivirus. In Virus Taxonomy: Eighth Report of the International Committee on Taxonomy of Viruses; Fauquet, C.M., Mayo, M.A., Maniloff, J., Desselberger, U., Ball, L.A., Eds.; Elsevier Academic Press: San Diego, CA, USA, 2005; pp. 534-542.

10. Isogai, M.; Uyeda, I.; Lee, B.C. Detection and assignment of proteins encoded by rice black streaked dwarf fijivirus S7, S8, S9 and S10. J. Gen. Virol. 1998, 79, 1487-1494. [CrossRef]

11. Firth, A.E.; Atkins, J.F. Analysis of the coding potential of the partially overlapping $3^{\prime}$ ORF in segment 5 of the plant fijiviruses. Virol. J. 2009, 6, 32. [CrossRef]

12. Liu, H.J.; Wei, C.H.; Zhong, Y.W.; Li, Y. Rice black-streaked dwarf virus minor core protein P8 is a nuclear dimeric protein and represses transcription in tobacco protoplasts. FEBS Lett. 2007, 581, 2534-2540. [CrossRef]

13. Zhang, H.; Li, L.; He, Y.; Qin, Q.; Chen, C.; Wei, Z.; Tan, X.; Xie, K.; Zhang, R.; Hong, G.; et al. Distinct modes of manipulation of rice auxin response factor OsARF17 by different plant RNA viruses for infection. Proc. Natl. Acad. Sci. USA 2020, 117, 9112-9121. [CrossRef]

14. Li, M.; Sun, X.; Di, D.; Zhang, A.; Qing, L.; Zhou, T.; Miao, H.; Fan, Z. Maize AKIN $\beta \gamma$ Proteins Interact with P8 of Rice Black Streaked Dwarf Virus and Inhibit Viral Infection. Viruses 2020, 12, 1387. [CrossRef] 
15. Otuka, A. Migration of rice planthoppers and their vectored re-emerging and novel rice viruses in East Asia. Front. Microbiol. 2013, 4, 309. [CrossRef]

16. Kang, B.-C.; Yeam, I.; Jahn, M.M. Genetics of Plant Virus Resistance. Annu. Rev. Phytopathol. 2005, 43, 581-621. [CrossRef]

17. Nicaise, V. Crop immunity against viruses: Outcomes and future challenges. Front. Plant Sci. 2014, 5, 660. [CrossRef] [PubMed]

18. Wang, A. Dissecting the Molecular Network of Virus-Plant Interactions: The Complex Roles of Host Factors. Annu. Rev. Phytopathol. 2015, 53, 45-66. [CrossRef] [PubMed]

19. Zaidi, S.S.-E.-A.; Tashkandi, M.; Mansoor, S.; Mahfouz, M.M. Engineering Plant Immunity: Using CRISPR/Cas9 to Generate Virus Resistance. Front. Plant Sci. 2016, 7, 1673. [CrossRef]

20. Sanfaçon, H. Plant Translation Factors and Virus Resistance. Viruses 2015, 7, 3392-3419. [CrossRef] [PubMed]

21. Shopan, J.; Lv, X.; Hu, Z.; Zhang, M.; Yang, J. Eukaryotic Translation Initiation Factors Shape RNA Viruses Resistance in Plants. Hortic. Plant J. 2020, 6, 81-88. [CrossRef]

22. Pyott, D.E.; Sheehan, E.; Molnar, A. Engineering of CRISPR/Cas9-mediated potyvirus resistance in transgene-free Arabidopsis plants. Mol. Plant Pathol. 2016, 17, 1276-1288. [CrossRef]

23. Chandrasekaran, J.; Brumin, M.; Wolf, D.; Leibman, D.; Klap, C.; Pearlsman, M.; Sherman, A.; Arazi, T.; Gal-On, A. Development of broad virus resistance in non-transgenic cucumber using CRISPR/Cas9 technology. Mol. Plant Pathol. 2016, 17, 1140-1153. [CrossRef]

24. Macovei, A.; Sevilla, N.R.; Cantos, C.; Jonson, G.B.; Slamet-Loedin, I.; Čermák, T.; Voytas, D.F.; Choi, I.R.; Chadha-Mohanty, P. Novel alleles of rice eIF4G generated by CRISPR/Cas9-targeted mutagenesis confer resistance to Rice tungro spherical virus. Plant Biotechnol. J. 2018, 16, 1918-1927. [CrossRef]

25. Wu, J.; Ni, Y.; Liu, H.; Rao, L.; Zhou, Y.; Zhou, X. Development and use of three monoclonal antibodies for the detection of rice black-streaked dwarf virus in field plants and planthopper vectors. Virol. J. 2013, 10, 114. [CrossRef] [PubMed]

26. Xing, H.-L.; Dong, L.; Wang, Z.-P.; Zhang, H.-Y.; Han, C.-Y.; Liu, B.; Wang, X.-C.; Chen, Q.-J. A CRISPR/Cas9 toolkit for multiplex genome editing in plants. BMC Plant Biol. 2014, 14, 1-12. [CrossRef] [PubMed]

27. Sun, F.; Fang, P.; Li, J.; Du, L.; Lan, Y.; Zhou, T.; Fan, Y.; Shen, W.; Zhou, Y. RNA-seq-based digital gene expression analysis reveals modification of host defense responses by rice stripe virus during disease symptom development in Arabidopsis. Virol. J. 2016, 13, 1-13. [CrossRef] [PubMed]

28. Livak, K.J.; Schmittgen, T.D. Analysis of relative gene expression data using real-time quantitative PCR and the $2(-\Delta \Delta \mathrm{Ct}) \mathrm{method}$. Methods 2001, 25, 402-408. [CrossRef]

29. Slykhuis, J.T. Virus and Virus-Like Diseases of Cereal Crops. Annu. Rev. Phytopathol. 1976, 14, 189-210. [CrossRef]

30. Sun, H.; Pu, J.; Chen, F.; Wang, J.; Han, Z. Multiple ATP-binding cassette transporters are involved in insecticide resistance in the small brown planthopper, Laodelphax striatellus. Insect Mol. Biol. 2017, 26, 343-355. [CrossRef]

31. Liu, Q.; Deng, S.; Liu, B.; Tao, Y.; Ai, H.; Liu, J.; Zhang, Y.; Zhao, Y.; Xu, M. A helitron-induced RabGDI $\alpha$ variant causes quantitative recessive resistance to maize rough dwarf disease. Nat. Commun. 2020, 11, 1-14. [CrossRef]

32. Khatodia, S.; Bhatotia, K.; Tuteja, N. Development of CRISPR/Cas9 mediated virus resistance in agriculturally important crops. Bioengineered 2017, 8, 274-279. [CrossRef]

33. Bastet, A.; Robaglia, C.; Gallois, J.-L. eIF4E Resistance: Natural Variation Should Guide Gene Editing. Trends Plant Sci. 2017, 22, 411-419. [CrossRef]

34. Nicaise, V.; Gallois, J.-L.; Chafiai, F.; Allen, L.M.; Schurdi-Levraud, V.; Browning, K.S.; Candresse, T.; Caranta, C.; Le Gall, O.; German-Retana, S. Coordinated and selective recruitment of eIF4E and eIF4G factors for potyvirus infection in Arabidopsis thaliana. FEBS Lett. 2007, 581, 1041-1046. [CrossRef]

35. Yoshii, M.; Nishikiori, M.; Tomita, K.; Yoshioka, N.; Kozuka, R.; Naito, S.; Ishikawa, M. The Arabidopsis Cucumovirus Multiplication 1 and 2 Loci Encode Translation Initiation Factors 4E and 4G. J. Virol. 2004, 78, 6102-6111. [CrossRef]

36. Albar, L.; Bangratz-Reyser, M.; Hébrard, E.; Ndjiondjop, M.N.; Jones, M.; Ghesquière, A. Mutations in the eIF(iso)4G translation initiation factor confer high resistance of rice to rice yellow mottle virus. Plant J. Cell Mol. Biol. 2010, 47, 417-426. [CrossRef]

37. Feng, Z.; Kang, H.; Li, M.; Zou, L.; Wang, X.; Zhao, J.; Wei, L.; Zhou, N.; Li, Q.; Lan, Y.; et al. Identification of new rice cultivars and resistance loci against rice black-streaked dwarf virus disease through genome-wide association study. Rice 2019, 12, 1-13. [CrossRef]

38. Xiao, S.; Wang, B.; Liu, Y.; Miao, T.; Zhang, H.; Wen, P.; He, J.; Huang, J.; Liu, D.; Qiu, Z.; et al. Genome-wide association study and linkage analysis on resistance to rice black-streaked dwarf virus disease. Mol. Breed. 2019, 39, 73. [CrossRef]

39. Calil, I.P.; Fontes, E.P.B. Plant immunity against viruses: Antiviral immune receptors in focus. Ann. Bot. 2017, 119, 711-723. [CrossRef]

40. Charron, C.; Nicolaï, M.; Gallois, J.L.; Robaglia, C.; Moury, B.; Palloix, A.; Caranta, C. Natural variation andfunctional analyses provide evidence for co-evolution between plant eIF4E and potyviral VPg. Plant J. 2008, 54, 56-68. [CrossRef]

41. Saha, S.; Mäkinen, K. Insights into the functions of eIF4E-binding motif of VPg in potato virus A infection. Viruses 2020, $12,197$. [CrossRef]

42. Hébrard, E.; Poulicard, N.; Gérard, C.; Traoré, O.; Wu, H.C.; Albar, L.; Fargette, D.; Bessin, Y.; Vignols, F. Direct interaction between the rice yellow mottle virus (RYMV) VPg and the central domain of the rice eIF(iso)4G1 factor correlates with rice susceptibility and RYMV virulence. Mol. Plant Microbe Interact. 2010, 23, 1506. [CrossRef] 